\title{
Unidirectional relationship between heroin self-administration and impulsive decision-making in rats
}

\author{
Maria C. Schippers • Rob Binnekade • \\ Anton N. M. Schoffelmeer • Tommy Pattij • \\ Taco J. De Vries
}

Received: 8 June 2011 / Accepted: 4 August 2011 / Published online: 2 September 2011

(C) The Author(s) 2011. This article is published with open access at Springerlink.com

\begin{abstract}
Rationale There is growing clinical evidence for a strong relationship between drug addiction and impulsivity. However, it is not fully clear whether impulsivity is a preexisting trait or a consequence of drug abuse. Recent observations in the animal models show that pre-existing levels of impulsivity predict cocaine and nicotine seeking. Whether such relationships also exist with respect to nonstimulant drugs is largely unknown.

Objective We studied the relationship between impulsive choice and vulnerability to heroin taking and seeking.

Materials and methods Rats were selected in the delayed reward task based on individual differences in impulsive choice. Subsequently, heroin intravenous self-administration behaviour was analysed, including acquisition of heroin intake, motivation, extinction and drug- and cue-induced reinstatement. Throughout the entire experiment, changes in impulsive choice were monitored weekly.

Results and discussion High impulsivity did not predict measures of heroin taking. Moreover, high impulsive rats did not differ from low impulsive rats in extinction rates or heroinand cue-induced reinstatement. However, both groups became more impulsive as heroin self-administration continued. During abstinence, impulsivity levels returned towards base-
\end{abstract}

Tommy Pattij and Taco J. De Vries equally contributed.

M. C. Schippers · R. Binnekade • A. N. M. Schoffelmeer •

T. Pattij $(\triangle) \cdot$ T. J. De Vries

Department of Anatomy and Neurosciences,

Neuroscience Campus Amsterdam,

Vrije Universiteit University Medical Center,

Van der Boechorststraat 7,

1081 BT Amsterdam, The Netherlands

e-mail: t.pattij@vumc.nl

M. C. Schippers

e-mail: r.schippers@vumc.nl line (pre-heroin) levels. Our results indicate that, in contrast to psychostimulants, impulsive choice does not predict vulnerability to heroin seeking and taking.

Conclusion These data implicate that different neural mechanisms may underlie the vulnerability to opiate and psychostimulant dependence. Moreover, our data suggest that elevated impulsivity levels as observed in heroindependent subjects are a consequence of heroin intake rather than a pre-existing vulnerability trait.

Keywords Impulsivity · Heroin · Opiate · Addiction .

Delayed reward task $\cdot$ Self-administration

\section{Introduction}

Drug addiction is a chronic relapsing disorder characterized by compulsive drug-taking and continuation of drug use despite the knowledge of negative consequences to the subjects' health status, their environment and society. The identification of risk factors for the aetiology of substance dependence is important for a better understanding of the underlying neural and psychological mechanisms and eventually the development and improvement of clinical interventions.

Accumulating evidence shows a strong relationship between drug addiction and impulsivity (de Wit 2009; MacKillop et al. 2011; Perry and Carroll 2008). Clinical studies have shown that drug-dependent subjects display elevated impulsivity scores on questionnaires and laboratory measures when compared with control subjects. This is found for different types of drugs, including heroin (Clark et al. 2006; Kirby et al. 1999; Madden et al. 1997; Odum et al. 2000), cocaine (Coffey et al. 2003) and nicotine (Bickel et al. 1999; Mitchell 1999). In addition, a high co-morbidity 
exists between substance abuse and psychiatric disorders characterized by compulsive or impulsive behaviour, such as ADHD (Wilson 2007). Although clinical data strongly suggest a relationship between impulsivity and drug addiction, it remains unclear whether impulsivity is a pre-existing trait or a consequence of drug abuse (Verdejo-García et al. 2008; Winstanley et al. 2010).

Importantly, impulsivity is a multifaceted construct and different forms of impulsivity can be dissociated at a behavioural, pharmacological and neuroanatomical level (Evenden 1999; Pattij and Vanderschuren 2008; Winstanley et al. 2006). Conceptually, impulsive behaviour can be divided into impulsive action, reflecting a lack of inhibitory response control, and impulsive decision-making, often operationalized as the insensitivity to delayed rewards and therefore a preference towards immediate, but objectively less beneficial rewards.

Preclinical work in rodents has revealed a relationship between pre-existing impulsivity traits and vulnerability to psychostimulant drug self-administration. Furthermore, these studies demonstrate a dissociation between different forms of impulsivity and their ability to predict distinct phases of drug self-administration. For instance, it has been shown that impulsive action is related to the initiation and maintenance of nicotine taking, whereas impulsive choice predicts a diminished capacity to inhibit drug-seeking when nicotine is not available and an increased relapse vulnerability upon re-exposure to nicotine-related cues (Diergaarde et al. 2008). Similarly, a strong relationship between impulsivity and cocaine taking and seeking has been reported by different laboratories (Anker et al. 2009; Belin et al. 2008; Dalley et al. 2007; Economidou et al. 2009; Perry et al. 2008), again with evidence for a distinct predictability of the different forms of impulsivity. Together these studies stress the notion that impulsive behaviour may predispose psychostimulant drug-taking and seeking.

As opposed to psychostimulants, the relationship between impulsive behaviour and the addictive properties of heroin has been studied in much less detail. Clinical data show a strong correlation between heroin use and impulsivity. For instance heroin-dependent subjects display elevated levels of impulsive choice behaviour in delayed discounting tasks compared to control subjects (Clark et al. 2006; Kirby et al. 1999; Kirby and Petry 2004; Madden et al. 1997). Recently, it has been shown that high impulsive rats, selected on an impulsive action task, did not differ in heroin intake compared to low impulsive rats (McNamara et al. 2010), suggesting that this form of impulsive behaviour does not predict heroin selfadministration behaviour. On the other hand, studies in Lewis and Fischer 344 rats have shown that these two rat strains differ in voluntary morphine intake and in impulsive choice behaviour (García-Lecumberri et al. 2011). This may indicate that impulsive choice is an underlying vulner- ability trait to opiate use, but the causal relationship was not tested directly.

The aim of the present study was to investigate whether a relationship exists between impulsive choice and different aspects of heroin taking and seeking. For this purpose, the rats were screened on impulsive choice, using the delayed reward task, and subsequently subjected to a heroin selfadministration paradigm to assess putative differences in the motivation for heroin taking, seeking and sensitivity to heroin-associated cues between high impulsive (HI) and low impulsive (LI) rats. In addition, during the course of the entire heroin self-administration protocol, impulsive choice was monitored to investigate whether levels of impulsive choice were altered by heroin intake and abstinence.

\section{Methods}

Animals

Forty-eight male Wistar rats, weighing between 260 and $290 \mathrm{~g}$ at the beginning of the experiments, were used for this experiment. The rats were housed in pairs in enriched Macrolon cages until implantation of an IV catheter and kept under standard conditions (lights on from 7 P.M. until 7 A.M.) with water available ad libitum during the entire experiment. From the start of the delayed reward task training until the end of the experiment, all the animals were food restricted and maintained at $85-95 \%$ of their free-feeding weight. The experiments were conducted during the dark phase of the light-dark cycle. All experiments were approved by the Animal Care Committee of the VU University and VU University Medical Center of Amsterdam.

\section{Design}

The experiment consisted of two phases as indicated in Fig. 1. In phase 1, the animals were trained on the delayed reward task and selected on high and low impulsive choice. In phase 2, the selected animals were implanted with a catheter in the jugular vein. After recovery, the animals were re-trained to baseline delayed reward task performance and subsequently trained to self-administer heroin. In addition, once weekly levels of impulsive choice were tested in the delayed reward task. On these days, the rats were first tested in the delayed reward task prior to heroin self-administration or extinction training. Testing of delayed reward performance lasted until 5 weeks after the reinstatement test. In this period, the rats were abstinent of heroin and any heroin-associated cues. Notably, nose pokes were used in the delayed reward task versus levers in the heroin self-administration paradigm, to prevent generalization between the tasks. 


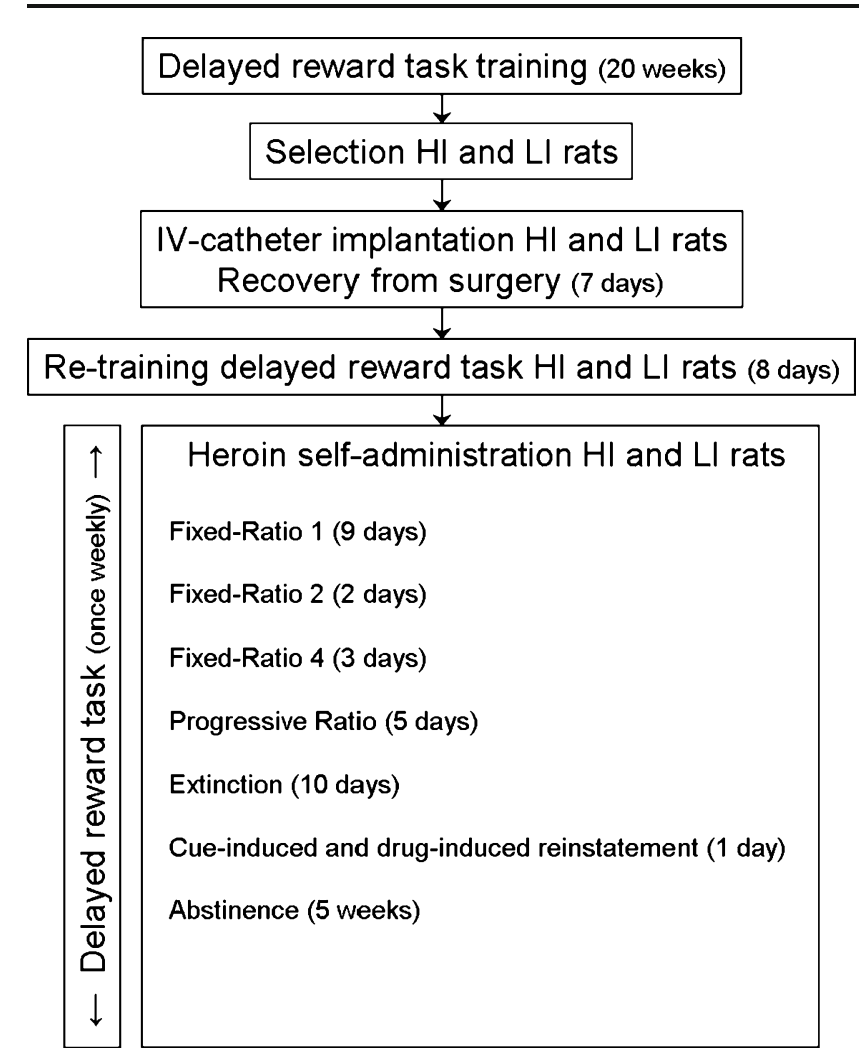

Fig. 1 Schematic diagram showing the experimental design of the present study. $H I$ high impulsive, $L I$ low impulsive

\section{Delayed reward task}

Apparatus The delayed reward task was conducted in operant chambers (Med Associates Inc., St. Albans, USA) in sound-attenuating ventilated cubicles. One wall contained five holes which could be illuminated and had an infrared beam for nose poke detection. For the purpose of the delayed reward task, only hole 2, 3 and 4 were used. On the opposite wall, a food magazine was situated, where the reward was delivered.

Delayed reward task The delayed reward paradigm as employed in our laboratory has been described more elaborately previously (van Gaalen et al. 2006). Briefly, in the final stages of training, a session was divided into 5 blocks of 12 trials, each block starting with 2 forced trials during which, after initiating the trial through a nose poke into the central unit, either the left unit or the right unit was illuminated in a counterbalanced fashion. In the next ten trials, the animals had a free choice and both the left and right unit were illuminated. Poking into one position resulted in the immediate delivery of a small reinforcer (one food pellet), whereas a nose poke into the other position resulted in the delivery of a large, but delayed, reinforcer (four food pellets). If an animal did not make a response during this choice phase within $10 \mathrm{~s}$, an intertrial interval was initiated and the trial was counted as an omission. The position associated with the small and large reinforcer was always the same for each individual, and counterbalanced for the group of rats. Delays for the large reinforcer progressively increased within a session per block of 12 trials as follows: $0,5,10,20$ and $40 \mathrm{~s}$. Responding into non-illuminated units during the test was recorded, but had no further programmed consequences. The behavioural measure to assess task performance, i.e. the percentage preference for the large reinforcer as a function of delay, was calculated as the number of choices for the large reinforcer choices/(number choices large+ small reinforcers $) \times 100$. In addition, we calculated the total number of omitted choice trials per block of ten trials within a session.

Selection of high and low impulsive animals

Labelling of the rats screened by means of the delayed reward task was based on the indifference point, the delay for which they switched their preference over to the immediate, small reward (i.e., the delay on which the preference for large reward $<50 \%$ [means of last four sessions of baseline]). This was calculated by the equation $V=A /(1+k D)$, where $V$ is the preference for the large reward after a delay of $D$ in seconds, $A$ is the preference for the large reward at $D=0 \mathrm{~s}$ and $k$ describes how rapidly $V$ declines with increasing delay (Mazur 2006). All high impulsive rats switched their preference at a delay of $\leq 10 \mathrm{~s}$, whereas all low impulsive rats switched at a delay of $\geq 20 \mathrm{~s}$. The previous work from our laboratory has shown that these selection criteria are stable and trait-like over time (Diergaarde et al. 2008; Diergaarde et al. 2009; Loos et al. 2010). Following stable baseline performance on the delayed reward task, 28 selected animals (14 high and 14 low impulsive) were implanted with a silicone catheter in the right jugular vein, under isoflurane anaesthesia. Following 1 week of recovery, the rats were trained in the delayed reward task for two more weeks to re-acquire stable baseline performance. Subsequently, the rats were trained to self-administer heroin and tested once weekly in the delayed reward task.

\section{Heroin self-administration}

Apparatus Self-administration of heroin was conducted in operant chambers (Med Associates Inc., St. Albans, USA) in sound-attenuating ventilated cubicles. On one wall, two levers were situated, of which one was a retractable, active lever, which was presented during a session. A cue light was situated above the active lever. All boxes were equipped with an auditory clicker. 
Acquisition The rats were trained to self-administer heroin (diacetylmorphine- $\mathrm{HCl}$, dissolved in $0.9 \%$ sterile saline, Slotervaart Hospital, The Netherlands) by pressing the active lever on a fixed-ratio-1 (FR1) schedule of reinforcement, whereby every lever press was reinforced with one infusion of $40 \mu \mathrm{l}$ heroin $(100 \mu \mathrm{g} / \mathrm{kg} /$ infusion) with a duration of $2 \mathrm{~s}$. The training consisted of $2.5 \mathrm{~h}$ daily sessions (Monday-Friday). Heroin infusions were accompanied by a 5 -s presentation of the cue light and five short auditory clicks of $0.8 \mathrm{~s}$ each. A house light was turned on during the entire session. Responses on the inactive lever were registered, but had no programmed consequences. After each drug infusion, a time-out period of $15 \mathrm{~s}$ was introduced, during which a lever press had no consequences. Responses on the active and inactive lever were registered, during availability of the drug and during the time-out period. After 9 days, the rats had acquired a stable response on FR1 and the FR schedule of reinforcement was increased to FR2 (2 days) and FR4 (3 days), meaning that every second or respectively fourth active lever press was reinforced.

Progressive ratio Following the acquisition phase, the animals were switched to a progressive ratio schedule of reinforcement. The number of lever presses on the active lever required for one heroin infusion was increased within one session according to the equation $5 \times e^{(0.25 \times[\text { Infusion number+3]) }}-5$ (Roberts and Bennett 1993). The infusions were accompanied by the cue light and five short clicks similar to acquisition. Each session lasted $4 \mathrm{~h}$.

Extinction Subsequently, responding for heroin was extinguished. For ten daily sessions of $60 \mathrm{~min}$, the animals were placed in the operant chamber, but lever pressing did not result in the delivery of heroin or presentation of the heroin-associated cues.

Cue- and drug-induced reinstatement Following extinction, a cue-induced reinstatement test was performed for $180 \mathrm{~min}$. The conditions were the same as during a FR4 session in the acquisition phase, with the exception that the animals did not receive heroin. The session started with the presentation of heroin-associated cues. Every fourth active lever press was accompanied by the cues. After 180 min responding was extinguished and a druginduced reinstatement test was performed by injecting $0.25 \mathrm{mg} / \mathrm{kg}$ heroin subcutaneously. After $15 \mathrm{~min}$, the rats were placed back in the operant test chamber for 60 min under the same conditions as during the cueinduced reinstatement test.

Abstinence After reinstatement testing, 5 weeks of abstinence followed, during which the rats were kept in their home-cages and were daily tested in the delayed reward task (Monday-Friday).

\section{Statistical analysis}

All data are presented as means \pm standard errors of the mean and were analysed using NCSS 2004 (Number Cruncher Statistical Systems, Kaysville, Utah, USA). Behavioural data from the delayed reward task and heroin self-administration were analysed using repeated measures analysis of variance (ANOVA), with impulsivity level (impulsivity) as between subjects factor for all experiments. In the case of statistical significance, further post hoc Newman-Keuls multiple comparison tests were performed. In the case of violation of homogeneity, tested with Mauchly's test of sphericity, corrected degrees of freedom and resulting more conservative $p$ values were used for subsequent analyses. Statistical significance was set at $p<0.05$.

\section{Results}

Impulsive choice selection

Out of the 48 rats that were trained in the delayed reward task, in total 14 high and 14 low impulsive rats were selected based on their preference for the large delayed reward and resulting indifference point. In total, three rats were excluded from all data analyses, since one LI animal had a clogged catheter, whereas two animals (one HI and one LI) did not achieve stable baseline performance on the delayed reward task after catheter implantation. This resulted in 13 high and 12 low impulsive rats for data analyses.

Both groups decreased their preference for the large reward in a delay-dependent manner and as expected impulsive choice differed between HI and LI. Repeated measures ANOVAs revealed a significant effect of delay $(F$ $(4,92)=514.34, p<0.001)$, impulsivity $(F(1,23)=68.56$, $p<0.001)$ and impulsivity $\times$ delay $(F(4,92)=31.35$, $p<0.001)$. Further ANOVAs indicated a group difference on all delays, except for the 0 -s delay ( $p<0.001$; Fig. 2). Total numbers of omissions to initiate a trial did not differ between impulsivity groups (HI, $6.36 \pm 1.08$; LI, $7.55 \pm 1.08$, $F(1,23)<1)$, indicating that there were no motivational differences between the groups. Response latencies for small rewards differed significantly, with HI responding faster (impulsivity, $F(1,23)=11.92, p=0.002$ ). Finally, the individual preference for the large delayed reward did not shift and remained stable after surgery (time, $F(1,23)=1.09$, NS). In addition, the number of omissions to initiate a trial was not influenced by surgery (time, $F(1,23)<1$ ). 


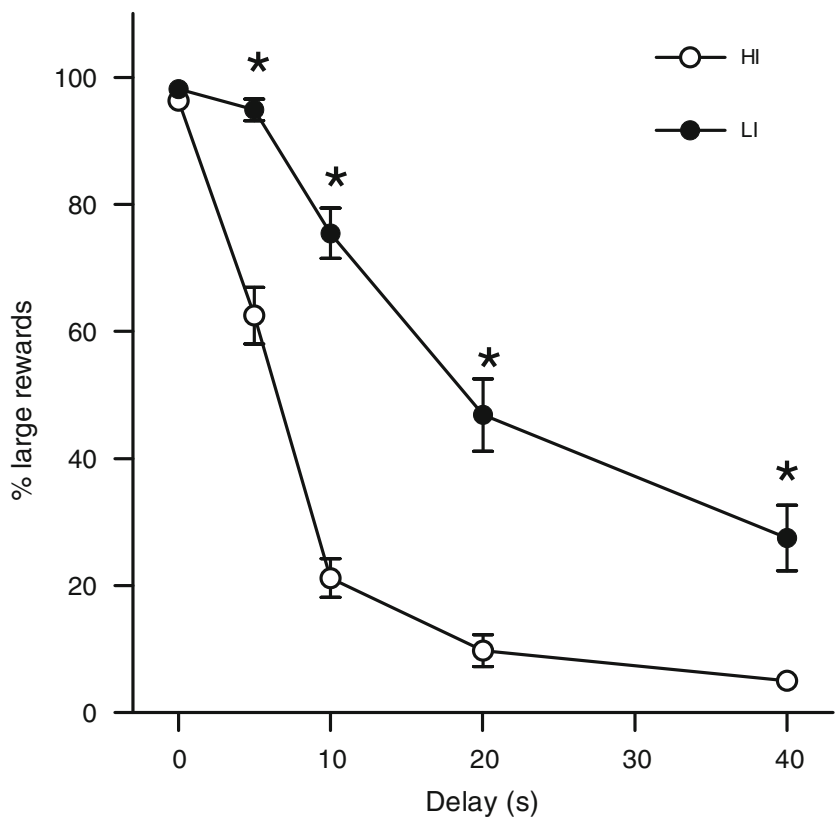

Fig. 2 Selection of high impulsive (HI) and low impulsive (LI) rats, based on their indifference points, calculated as an average of the last 4 days of a stable baseline period on the delayed reward task. HI had a significant lower preference for the large reward compared to LI on all but the 0 s delay. $H I$ high impulsive $(n=13), L I$ low impulsive $(n=12)$. ${ }^{*} p<0.001$

\section{Heroin self-administration}

All rats readily acquired heroin self-administration during nine daily sessions on a FR1 self-administration schedule $($ session $\times$ lever, $F(8,151)=5.50, p<0.001)$. HI and LI rats did not differ in the amount of lever pressing on either the FR1, FR2 or FR4 schedule (impulsivity $\times$ session FR1 (9 sessions), $F(8,173)<1$; FR2 (2 sessions), $F(1,21)=1.22$, NS; FR4 (3 sessions), $F(2,44)<1$; impulsivity $\times$ lever FR1, $F(1,23)<1$; FR2, $F(1,20)=1.21$, NS; FR4, $F(1,21)<1$; Fig. 3a). Similarly, no difference was found in total heroin intake over the entire self-administration period (HI, $5.09 \pm$ $0.43 \mathrm{mg}$; LI, $6.28 \pm 0.99 \mathrm{mg} ; F(1,23)=1.79$, NS).

After acquisition of heroin self-administration, the rats were subjected to a within-session PR schedule. The active lever presses were significantly higher compared to inactive lever presses (lever, $F(1,21)=16.88, p<0.001$ ). The number of active and inactive lever presses on a PR schedule was not differentially affected by impulsivity levels (impulsivity $\times$ lever, $F(1,21)<1$; Fig. 3b). Moreover, HI and LI rats reached a similar breaking point (HI, 75.23 \pm 18.93 ; LI, 58.00 \pm 17.83 , $F(1,23)<1)$.

During ten subsequent sessions, heroin self-administration was extinguished and all the rats rapidly decreased responding on the active lever in absence of heroin and heroin-associated stimuli (session, $F(9,207)=21.13, p<0.001$; lever, $F(1,23)=$ $35.40, p<0.001$; session $\times$ lever, $F(9,201)=25.67, p<0.001)$. During the extinction phase responding of $\mathrm{HI}$ rats did not differ from LI rats (impulsivity, $F(1,23)<1$; impulsivity $\times$ session, $F(9,207)<1$; Fig. 4a).

The presentation of heroin-associated cues and the ability to respond for presentation of these cues on a FR4 schedule reinstated responding on the previously active lever compared to the average of the last three extinction days (extinction compared with the total of first hour of reinstatement testsession, $F(1,18)=169.43, p<0.001$; lever, $F(1,26)=48.02$, $p<0.001$; session $\times$ lever, $F(1,18)=63.31, p<0.001)$. HI rats did not differ in number of lever presses compared to LI rats (impulsivity, $F(1,26)=1.68$, NS; Fig. 4b). After 3 h, responding on the previously active lever was extinguished (time, $F(35,770)=8.61, p<0.001 ;$ lever, $F(1,22)=29.79$, $p<0.001$; time $\times$ lever, $F(35,770)=4.46, p<0.001)$, with no differences between HI and LI rats (impulsivity, $F(1,22)<1$ ). Subsequently, all rats received a subcutaneous injection with heroin and were re-tested in presence of heroin-associated cues after $15 \mathrm{~min}$. The injection of heroin robustly reinstated active lever pressing (session, $F(2,36)=15.07, p<0.001$; lever,
Fig. 3 a Number of active and inactive lever presses for heroin during 150 min selfadministration sessions on FR1, FR2 and FR4 schedule for HI and LI. b Total number of active and inactive lever presses during a progressive ratio schedule of heroine reinforcement in $\mathrm{HI}$ and LI rats a

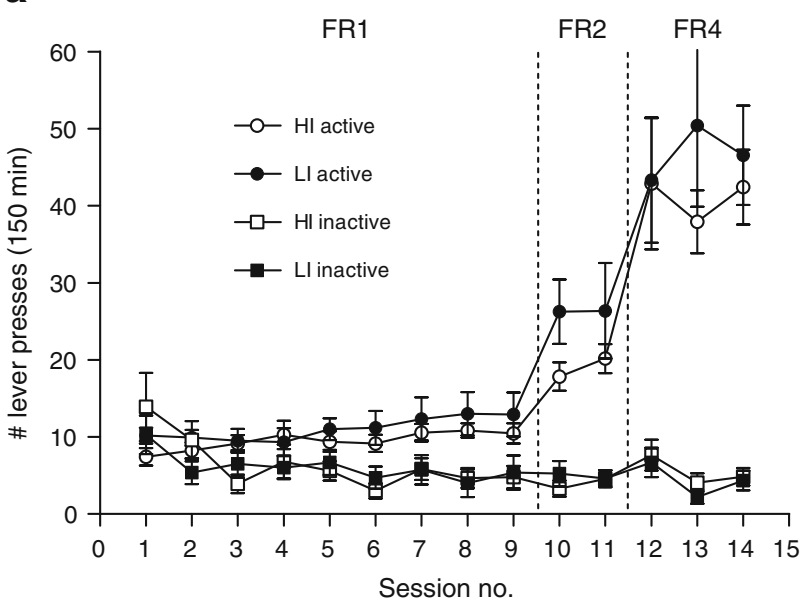

b

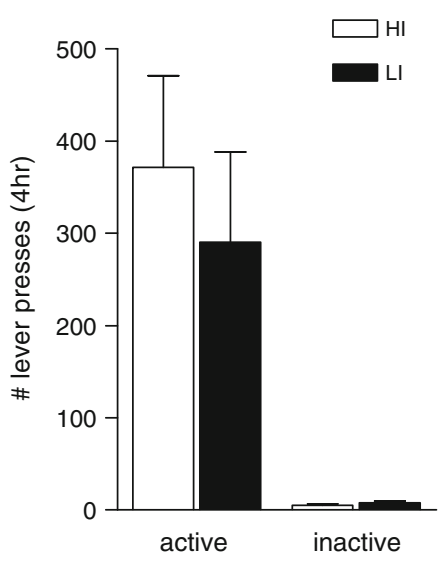


Fig. 4 a Number of active and inactive lever presses during the extinction of heroine selfadministration in $\mathrm{HI}$ and LI rats. b Number of total active and inactive lever presses during $60 \mathrm{~min}$ of cue-induced and 60 min of drug-induced reinstatement, compared to the average of the last three extinction sessions for $\mathrm{HI}$ and $\mathrm{LI}$
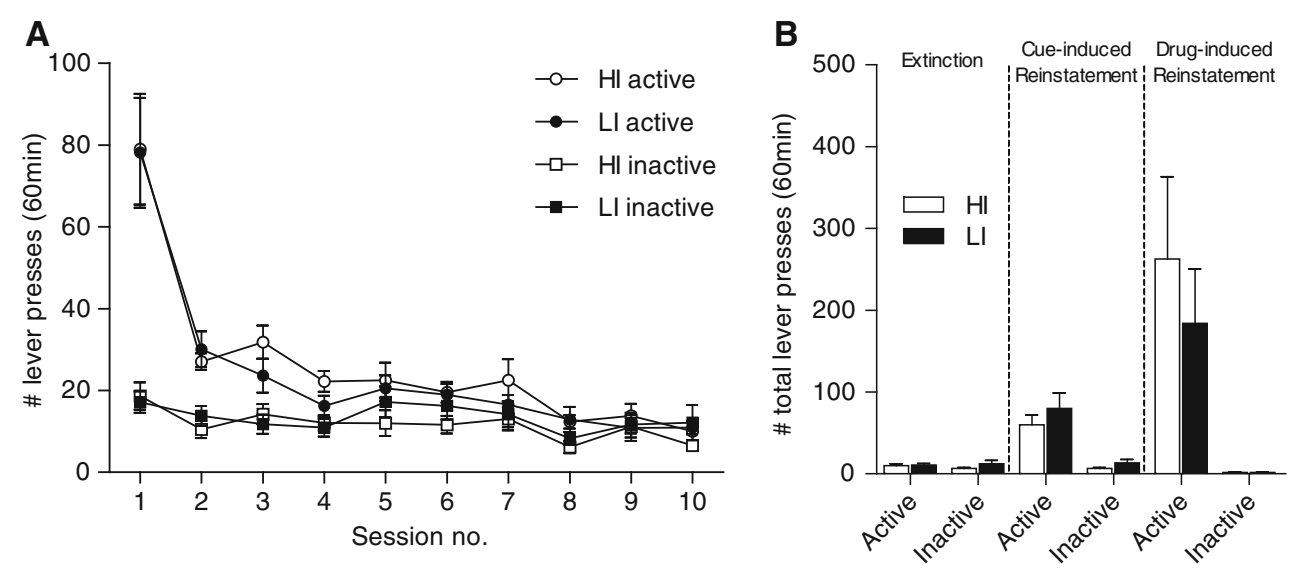

$F(1,26)=28.28, p<0.001$; session $\times$ lever, $F(2,36)=17.54$, $p<0.001)$, with no differential effects in HI compared to LI rats (impulsivity, $F(1,26)<1$; Fig. $4 b$ ).

Impulsive choice during and after heroin self-administration

During heroin self-administration, extinction and abstinence, the rats were trained weekly in the delayed reward task to assess impulsive choice. The analyses of indifference points revealed that impulsive choice was significantly increased by heroin self-administration (session, $F(7,153)=3.16, p=0.016)$. A lack of significant interaction between session and impulsivity indicated that this increment in impulsive choice during heroin selfadministration was independent of pre-heroin impulsivity levels (impulsivity $\times$ session, $F(7,153)=1.02$, NS; Fig. 5a). This was supported by additional analyses of the percentage change in indifference point compared to pre-surgery levels (impulsivity, $F(1,23)=1.63$, NS; session, $F(7,159)=4.47, p=0.004$; impulsivity $\times$ session, $F$ $(7,159)<1)$. Post hoc comparisons revealed a significant decreased indifference point during the FR2 and progressive ratio phase of heroin self-administration, indicating increased impulsive choice $(p<0.05$; Fig. $5 b)$. In addition, the complete data set for each time-point of the delayed reward task during different stages of heroin selfadministration is shown in Table 1. Overall, heroin exposure influenced the percentage large reward for every delay and this occurred to the same extent in HI and LI rats (session, $F(7,161)=3.65, p=0.001$; impulsivity $\times$ session, $F(7,161)=1.03$, NS; impulsivity $\times$ delay, $F(4,92)=$ $17.62, p<0.001)$. Post hoc comparisons revealed a significant decreased preference for the large reward during the FR2 and progressive ratio phase of heroin selfadministration compared to baseline, indicating increased impulsive choice for HI and LI rats $(p<0.05)$. Further in-depth analyses revealed no effect of heroin exposure on the percentage preference for the large reward
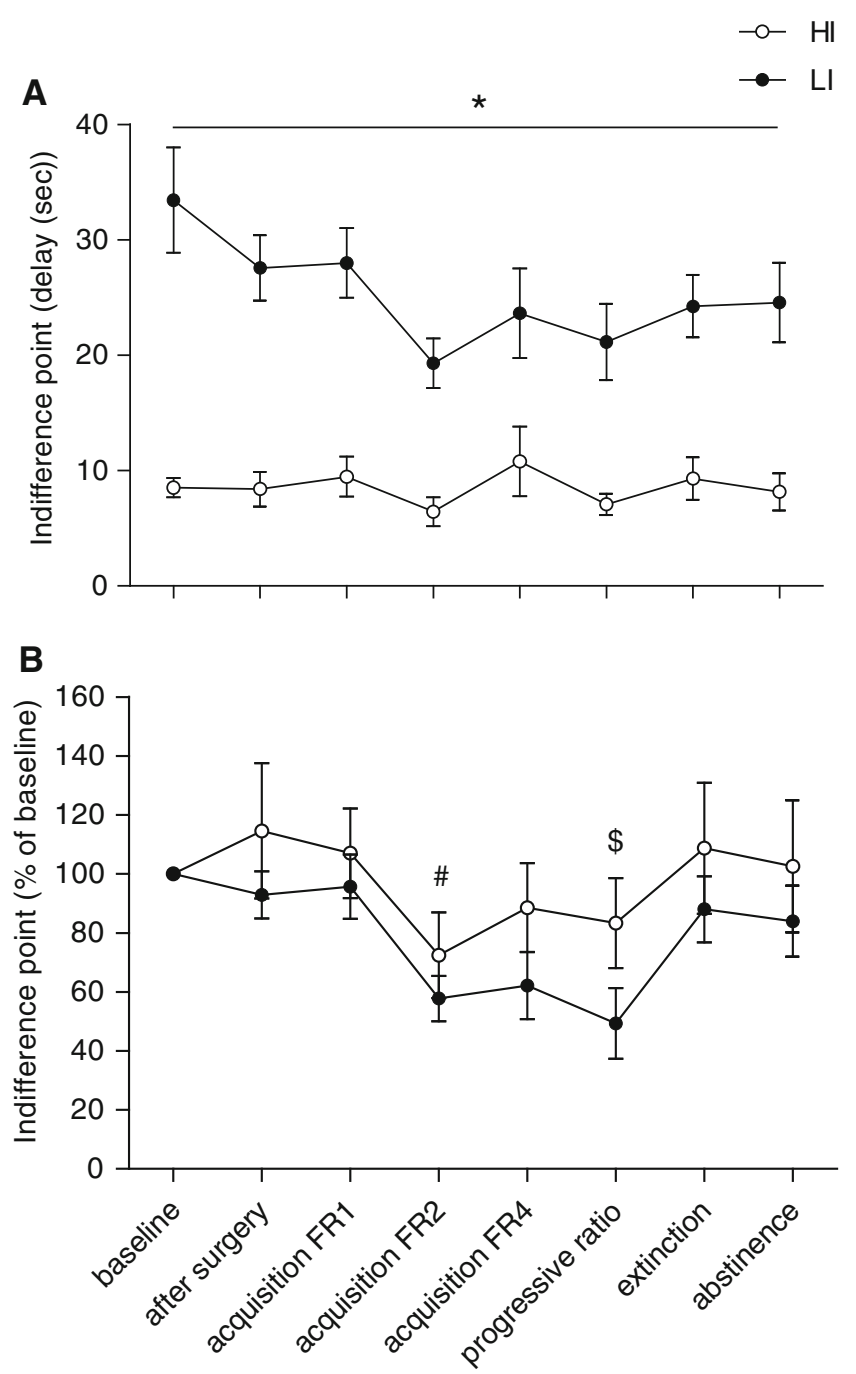

Fig. 5 Indifference point as indication of impulsive behaviour as an average of the last 4 days of baseline, 3 days of stable baseline after IV canulation, during FR1, FR2, FR4, progressive ratio, extinction and the average of the last 3 days after 5 weeks of abstinence. Data are shown for $\mathrm{HI}$ and LI, expressed as a absolute data and $\mathbf{b}$ percentage of baseline. *HI vs LI $p<0.001$; \#FR2 vs baseline, after surgery, extinction; \$PR vs baseline, after surgery, extinction, abstinence $p<0.05$ 
Table 1 Behavioural performance of HI and LI rats in the delayed reward task during different stages of heroin selfadministration
Preference for the large reward, expressed as percentage of total choice for the large reward $( \pm$ SEM), for every delay in the delayed reward task tested during different time-points of heroin self-administration and during extinction and abstinence for $\mathrm{HI}$ and $\mathrm{LI}$ rats

\begin{tabular}{|c|c|c|c|c|c|c|}
\hline \multirow[t]{2}{*}{ Session } & & \multicolumn{5}{|l|}{ Delay (s) } \\
\hline & & 0 & 5 & 10 & 20 & 40 \\
\hline \multirow[t]{2}{*}{ Baseline } & $\mathrm{HI}$ & $96.04 \pm 1.12$ & $63.53 \pm 4.63$ & $22.11 \pm 3.10$ & $10.01 \pm 2.68$ & $4.73 \pm 1.05$ \\
\hline & LI & $97.89 \pm 0.88$ & $94.19 \pm 1.93$ & $72.81 \pm 4.12$ & $41.74 \pm 5.07$ & $22.49 \pm 2.52$ \\
\hline \multirow[t]{2}{*}{ After surgery } & $\mathrm{HI}$ & $91.77 \pm 4.29$ & $60.24 \pm 6.57$ & $22.17 \pm 5.85$ & $10.07 \pm 2.43$ & $6.93 \pm 1.62$ \\
\hline & LI & $96.67 \pm 1.30$ & $89.14 \pm 3.29$ & $68.45 \pm 4.92$ & $37.39 \pm 5.17$ & $19.10 \pm 3.94$ \\
\hline \multirow[t]{2}{*}{ Acquisition FR1 } & $\mathrm{HI}$ & $89.06 \pm 4.60$ & $62.16 \pm 6.17$ & $25.74 \pm 6.25$ & $7.41 \pm 2.64$ & $4.53 \pm 1.65$ \\
\hline & LI & $97.87 \pm 0.98$ & $89.79 \pm 3.18$ & $64.09 \pm 6.20$ & $37.01 \pm 7.34$ & $18.93 \pm 3.44$ \\
\hline \multirow[t]{2}{*}{ Acquisition FR2 } & $\mathrm{HI}$ & $95.13 \pm 1.98$ & $51.43 \pm 8.55$ & $14.87 \pm 4.11$ & $4.87 \pm 2.19$ & $2.59 \pm 1.37$ \\
\hline & LI & $98.33 \pm 1.12$ & $86.59 \pm 3.60$ & $53.75 \pm 8.98$ & $20.51 \pm 5.35$ & $9.95 \pm 3.45$ \\
\hline \multirow[t]{2}{*}{ Acquisition FR4 } & HI & $94.00 \pm 2.99$ & $60.13 \pm 7.94$ & $26.73 \pm 9.16$ & $12.55 \pm 6.02$ & $2.91 \pm 1.58$ \\
\hline & LI & $99.17 \pm 0.83$ & $73.31 \pm 8.92$ & $55.02 \pm 13.28$ & $32.80 \pm 4.36$ & $16.82 \pm 5.19$ \\
\hline \multirow[t]{2}{*}{ Progressive ratio } & $\mathrm{HI}$ & $91.88 \pm 3.87$ & $62.05 \pm 6.48$ & $12.82 \pm 5.13$ & $5.92 \pm 2.03$ & $2.39 \pm 1.70$ \\
\hline & LI & $96.46 \pm 1.52$ & $83.98 \pm 5.63$ & $59.27 \pm 10.34$ & $31.37 \pm 6.44$ & $5.72 \pm 2.63$ \\
\hline \multirow[t]{2}{*}{ Extinction } & HI & $96.88 \pm 0.91$ & $60.72 \pm 7.41$ & $24.58 \pm 6.42$ & $7.03 \pm 2.56$ & $6.09 \pm 2.59$ \\
\hline & LI & $100.00 \pm 0.00$ & $92.32 \pm 2.78$ & $67.49 \pm 6.79$ & $27.92 \pm 5.53$ & $13.97 \pm 3.42$ \\
\hline \multirow[t]{2}{*}{ Abstinence } & $\mathrm{HI}$ & $93.18 \pm 1.95$ & $60.91 \pm 7.71$ & $17.83 \pm 6.34$ & $5.14 \pm 1.64$ & $3.29 \pm 1.55$ \\
\hline & LI & $97.35 \pm 1.11$ & $91.30 \pm 3.23$ & $61.09 \pm 8.00$ & $23.10 \pm 6.12$ & $13.96 \pm 4.10$ \\
\hline
\end{tabular}

on the 0 -s delay, suggesting that heroin exposure did not influence motivational aspects in HI and LI rats (session, $F(7,161)=1.25$, NS; session $\times$ impulsivity, $F(7,161)<1)$.

Significant effects between HI and LI rats were found on the number of omissions to start a trial, omissions to make a choice and response latency for a small reward, with higher numbers for LI rats (omissions start - impulsivity, $F(1,23)=$ 4.85, $p=0.038$; omissions choice - impulsivity, $F(1,23)=$ 6.42, $p=0.019$; response latency-impulsivity, $F(1,23)=$ $7.55, p=0.011)$. During the course of the entire selfadministration protocol, changes were observed for the number of omissions to start a trial (session, $F(7,161)=$ 2.99, $p=0.027$ ) and the number of omissions to make choice (session, $F(7,161)=3.09, p=0.025$ ), in the same manner for $\mathrm{HI}$ and LI rats (session $\times$ impulsivity omissions start, $F$ $(7,161)<1$; omissions choice, $F(7,161)<1)$. Post hoc comparisons showed that omissions were significantly increased only during the progressive ratio phase of heroin selfadministration as compared to omissions made in the delayed reward task monitored during pre-surgery, after surgery, FR4, extinction and abstinence $(p<0.05)$. Notably, the differences were very small (omissions start PR, 1.97 vs after surgery, 1.17; omissions choice PR, 1.13 vs after surgery, 0.93).

\section{Discussion}

This study examined the relationship between impulsive choice and heroin taking and seeking. Our data indicate that heroin intake increases impulsive choice over time and demonstrate that impulsive choice is altered by volitional heroin self-administration independent of pre-existing individual impulsivity levels. This decrease in self-controlled choice was transient and only observed during the heroin selfadministration phase. During extinction and abstinence, these elevated impulsivity levels returned to baseline. This observation suggests that the elevated impulsive choice observed in heroin-dependent subjects (Clark et al. 2006; Kirby et al. 1999; Madden et al. 1997; Odum et al. 2000) occurs as a consequence of heroin intake, rather than being a vulnerability trait.

Secondly, the results revealed no effect of pre-existing levels of impulsive choice on acquisition of heroin selfadministration, nor on the motivation to self-administer heroin. Furthermore, the rate of extinction and the sensitivity to relapse to drug seeking provoked by heroin-related cues or heroin priming was not influenced by trait impulsivity.

Impulsive choice does not predict vulnerability to heroin taking and seeking

Our observations are in striking contrast with previous findings showing that impulsive action and impulsive choice predict vulnerability to different phases of (volitional) self-administration of the stimulants nicotine and cocaine. For instance, impulsive choice appeared to be primarily related to the persistence and reinstatement of nicotine seeking during abstinence, whereas impulsive action was associated with the number of nicotine infusions and the motivation to work for nicotine reward (Diergaarde 
et al. 2008). A similar relationship has been reported between pre-existing impulsivity and cocaine selfadministration (Belin et al. 2008; Economidou et al. 2009). Furthermore, high impulsive rats showed a greater tendency to escalate in long-access cocaine selfadministration protocols (Anker et al. 2009; Dalley et al. 2007; Perry et al. 2008), which is suggested to indicate compulsive drug-seeking rather than controlled drug-taking (Ahmed and Koob 1998). Interestingly, similar to the main findings of the present study, individual variability in impulsive action also did not predict short-access and longaccess heroin intake in rats (McNamara et al. 2010).

Collectively, this might imply that the aetiology of heroin addiction differs, at least in part, from the aetiology and the risk factors involved in psychostimulant addiction. A possible neurobiological explanation may relate to differential involvement of the mesolimbic dopamine system. Individual differences in impulsive behaviour are associated with differences in the sensitivity of the dopamine system. For instance, drug-naive HI rats screened for either impulsive action or impulsive choice displayed differences in the releasability of dopamine in various mesocorticolimbic brain regions as compared to LI animals, most notably in the medial prefrontal cortex and nucleus accumbens shell region (Diergaarde et al. 2008). Moreover, trait impulsive action is associated with a decreased availability of dopamine D2/3 receptors in the ventral striatum of $\mathrm{HI}$ animals (Dalley et al. 2007). Interestingly, recent neuroimaging data in humans support this notion and reveal a correlation between trait impulsivity and dopamine receptor availability in the ventral striatum (Buckholtz et al. 2010). Accordingly, pharmacological studies in rats revealed an important role of mesolimbic dopamine in impulsive behaviour (Pattij and Vanderschuren 2008; Winstanley et al. 2006). Since both cocaine and nicotine self-administration strongly depend on the release of dopamine (Di Chiara 2000; Willuhn et al. 2010), vulnerability to psychostimulant addiction may be related to a pre-existing difference in the tuning of the dopamine system.

Consequently, it is conceivable that the development and persistence of heroin self-administration are largely dopamine independent. Indeed, there is strong evidence that non-dopaminergic pathways are involved in the reinforcing and rewarding effects of opiates (Pierce and Kumaresan 2006). For example, the destruction of dopamine terminals in the nucleus accumbens attenuated cocaine but not heroin self-administration in rats (Gerrits and van Ree 1996; Pettit et al. 1984). Moreover, pre-treatment with the D2/D3 antagonist sulpiride does not affect intra-accumbal morphine self-administration (David et al. 2002). In addition, conditioned place preference for the opiate morphine remained unaltered by blockade of dopamine receptors in rats (Nader and van der Kooy 1997). Collectively, these studies suggest that the rewarding and reinforcing properties of heroin do not rely on the integrity of the mesolimbic dopamine system, which may explain the lack of a relationship between trait impulsivity and the vulnerability to heroin taking and seeking as observed here in the current study. Nonetheless, it is important to remark at this point that a dose-response curve of heroin was not included in the current experiments, which could provide further insights into the sensitivity towards the incentive properties of heroin in $\mathrm{HI}$ and LI rats.

Heroin intake increases impulsive choice

Various studies have shown elevated levels of impulsive behaviour in opiate dependent subjects. In general, opiate abusers appear to be primarily impaired on behavioural measures of decision-making (MacKillop et al. 2011; Perry and Carroll 2008; Verdejo-García et al. 2008). For instance, higher discounting rates were found in heroin-dependent subjects for monetary rewards (Kirby et al. 1999; Madden et al. 1997; Odum et al. 2000) and were correlated to selfreported impulsivity (Kirby et al. 1999; Madden et al. 1997). Furthermore, heroin-dependent subjects discounted delayed heroin significantly more than delayed monetary rewards (Madden et al. 1997).

In this respect, our present findings indicate that elevated impulsive choice is a consequence rather than a cause of heroin intake. In support of this notion, we observed a significant effect of heroin exposure on impulsive choice during our measurements of heroin taking and seeking. Thus, impulsivity increased during the course of heroin self-administration, i.e. during cumulative heroin consumption, and decreased again during extinction of heroin self-administration and 5 weeks of abstinence. Moreover, the observed changes in impulsive choice were independent of pre-existing individual impulsivity levels.

It is of interest to note that different acute effects of opiates on impulsivity have been reported. In preclinical studies, acute peripheral administration of the $\mu$-opioid receptor agonist morphine appeared to increase both impulsive action and impulsive choice (Kieres et al. 2004; Pattij et al. 2009). These findings are paralleled by the observation that a $\delta$-opioid receptor agonist SNC80 also enhanced measures of impulsive action (Befort et al. 2011). However, contrasting these data acute intravenous morphine administration failed to change impulsive choice in two different rat strains (García-Lecumberri et al. 2011). In addition, in healthy volunteers, pharmacological challenges with the opioid agonist oxycodone did not increase laboratory measures of impulsivity (Zacny and de Wit 2009). In contrast to our observations, Harty et al. reported that nine consecutive intraperitoneal 
injections of heroin had no significant effect on delayed reward task performance (Harty et al. 2011). It should be noted, however, that active and passive administration of heroin can lead to differential drug-induced neuroadaptations in mesocorticolimbic structures (Jacobs et al. 2003; Jacobs et al. 2002). In this respect, recent observations revealed that heroin-self-administration induces various molecular and cellular alterations in the prefrontal cortex (Van den Oever et al. 2008; Van den Oever et al. 2010), a brain area that, together with the nucleus accumbens, is known to be critically involved in drug addiction and impulsive behaviour (Koob and Volkow 2009; Pattij and Vanderschuren 2008).

In the current study, the impulsive choice returned to baseline levels during extinction and the following 5 weeks of abstinence. This finding is in agreement with previous data on impulsive action in rats, showing that impulsive action after $24 \mathrm{~h}$ and 3 weeks of withdrawal of heroin selfadministration was comparable to pre-heroin selfadministration levels of impulsivity (Dalley et al. 2005; McNamara et al. 2010). Interestingly, clinically former opiate dependents did not seem to differ from current opiate users in their performance on laboratory measures of impulsivity (Clark et al. 2006). This might suggest that more prolonged exposure to heroin may lead to more longlasting elevation of impulsivity even after prolonged periods of abstinence similar to other executive dysfunctions (Ersche et al. 2006). Obviously, unfavourable decision-making could diminish the cognitive abilities to reduce heroine intake and thereby contribute to the persistence of heroin addiction.

\section{Conclusion}

Taken together, our data provide evidence that volitional intake of heroin promotes impulsive decision-making in a delayed reward task in rats. On the other hand and in contrast to earlier observations with psychostimulants, pre-existing levels of impulsive choice did not predict individual differences in the sensitivity towards various aspects of heroin taking and seeking. Our findings extend those of McNamara and co-workers (2010), and collectively indicate that two distinct forms of impulsive behaviour, namely impulsive action (McNamara et al. 2010) and impulsive choice (present study), do not predict vulnerability to different aspects of volitional heroin selfadministration. This indicates that different behavioural traits may underlie the vulnerability to opiate and psychostimulant addiction. As such, our data imply that elevated impulsivity in heroin-dependent subjects is a consequence of heroin intake rather than a pre-existing vulnerability trait. In terms of clinical interventions in drug dependence, this would implicate that targeting impulsivity might be more effective in stimulant than in opiate dependent individuals.

Acknowledgements This research was funded by ZonMW grant 31160204 .

Open Access This article is distributed under the terms of the Creative Commons Attribution Noncommercial License which permits any noncommercial use, distribution, and reproduction in any medium, provided the original author(s) and source are credited.

\section{References}

Ahmed SH, Koob GF (1998) Transition from moderate to excessive drug intake: change in hedonic set point. Science 282:298-300

Anker JJ, Perry JL, Gliddon LA, Carroll ME (2009) Impulsivity predicts the escalation of cocaine self-administration in rats. Pharmacol Biochem Behav 93:343-348

Befort K, Mahoney M, Chow C, Hayton S, Kieffer B, Olmstead M (2011) Effects of delta opioid receptors activation on a response inhibition task in rats. Psychopharmacology (Berl) 214:967-976

Belin D, Mar AC, Dalley JW, Robbins TW, Everitt BJ (2008) High impulsivity predicts the switch to compulsive cocaine-taking. Science 320:1352-1355

Bickel WK, Odum AL, Madden GJ (1999) Impulsivity and cigarette smoking: delay discounting in current, never, and ex-smokers. Psychopharmacology (Berl) 146:447-454

Buckholtz JW, Treadway MT, Cowan RL, Woodward ND, Li R, Ansari MS, Baldwin RM, Schwartzman AN, Shelby ES, Smith CE, Kessler RM, Zald DH (2010) Dopaminergic network differences in human impulsivity. Science 329:532

Clark L, Robbins TW, Ersche KD, Sahakian BJ (2006) Reflection impulsivity in current and former substance users. Biol Psychiatry 60:515-522

Coffey SF, Gudleski GD, Saladin ME, Brady KT (2003) Impulsivity and rapid discounting of delayed hypothetical rewards in cocainedependent individuals. Exp Clin Psychopharmacol 11:18-25

Dalley JW, Laane K, Pena Y, Theobald DE, Everitt BJ, Robbins TW (2005) Attentional and motivational deficits in rats withdrawn from intravenous self-administration of cocaine or heroin. Psychopharmacology (Berl) 182:579-587

Dalley JW, Fryer TD, Brichard L, Robinson ES, Theobald DE, Laane K, Pena Y, Murphy ER, Shah Y, Probst K, Abakumova I, Aigbirhio FI, Richards HK, Hong Y, Baron JC, Everitt BJ, Robbins TW (2007) Nucleus accumbens D2/3 receptors predict trait impulsivity and cocaine reinforcement. Science 315:12671270

David V, Durkin T, Cazala P (2002) Differential effects of the dopamine D2/D3 receptor antagonist sulpiride on self-administration of morphine into the ventral tegmental area or the nucleus accumbens. Psychopharmacology (Berl) 160:307-317

de Wit H (2009) Impulsivity as a determinant and consequence of drug use: a review of underlying processes. Addict Biol $14: 22-31$

Di Chiara G (2000) Role of dopamine in the behavioural actions of nicotine related to addiction. Eur J Pharmacol 393:295-314

Diergaarde L, Pattij T, Poortvliet I, Hogenboom F, de Vries W, Schoffelmeer AN, De Vries TJ (2008) Impulsive choice and impulsive action predict vulnerability to distinct stages of nicotine seeking in rats. Biol Psychiatry 63:301-308

Diergaarde L, Pattij T, Nawijn L, Schoffelmeer AN, De Vries TJ (2009) Trait impulsivity predicts escalation of sucrose seeking 
and hypersensitivity to sucrose-associated stimuli. Behav Neurosci 123:794-803

Economidou D, Pelloux Y, Robbins TW, Dalley JW, Everitt BJ (2009) High impulsivity predicts relapse to cocaine-seeking after punishment-induced abstinence. Biol Psychiatry 65:851-856

Ersche KD, Clark L, London M, Robbins TW, Sahakian BJ (2006) Profile of executive and memory function associated with amphetamine and opiate dependence. Neuropsychopharmacology 31:1036-1047

Evenden JL (1999) Varieties of impulsivity. Psychopharmacology (Berl) 146:348-361

García-Lecumberri C, Torres I, Martín S, Crespo JA, Miguéns M, Nicanor C, Higuera-Matas A, Ambrosio E (2011) Strain differences in the dose-response relationship for morphine selfadministration and impulsive choice between Lewis and Fischer 344 rats. J Psychopharmacol 25:783-791

Gerrits MAFM, van Ree JM (1996) Effect of nucleus accumbens dopamine depletion on motivational aspects involved in initiation of cocaine and heroin self-administration in rats. Brain Res 713:114-124

Harty SC, Whaley JE, Halperin JM, Ranaldi R (2011) Impulsive choice, as measured in a delay discounting paradigm, remains stable after chronic heroin administration. Pharmacol Biochem Behav 98:337-340

Jacobs EH, Spijker S, Verhoog CW, Kamprath K, De Vries TJ, Smit AB, Schoffelmeer ANM (2002) Active heroin administration induces specific genomic responses in the nucleus accumbens shell. FASEB J 16:1961-1963

Jacobs EH, Smit AB, De Vries TJ, Schoffelmeer ANM (2003) Neuroadaptive effects of active versus passive drug administration in addiction research. Trends Pharmacol Sci 24:566-573

Kieres AK, Hausknecht KA, Farrar AM, Acheson A, de Wit H, Richards JB (2004) Effects of morphine and naltrexone on impulsive decision making in rats. Psychopharmacology (Berl) 173:167-174

Kirby KN, Petry NM (2004) Heroin and cocaine abusers have higher discount rates for delayed rewards than alcoholics or non-drugusing controls. Addiction 99:461-471

Kirby KN, Petry NM, Bickel WK (1999) Heroin addicts have higher discount rates for delayed rewards than non-drug-using controls. J Exp Psychol Gen 128:78-87

Koob GF, Volkow ND (2009) Neurocircuitry of addiction. Neuropsychopharmacology 35:217-238

Loos M, Pattij T, Janssen MC, Counotte DS, Schoffelmeer AN, Smit AB, Spijker S, van Gaalen MM (2010) Dopamine receptor D1/ D5 gene expression in the medial prefrontal cortex predicts impulsive choice in rats. Cereb Cortex 20:1064-1070

MacKillop J, Amlung M, Few L, Ray L, Sweet L, Munafò M (2011) Delayed reward discounting and addictive behavior: a metaanalysis. Psychopharmacology (Berl) 216:305-321

Madden GJ, Petry NM, Badger GJ, Bickel WK (1997) Impulsive and self-control choices in opioid-dependent patients and non-drugusing control participants: drug and monetary rewards. Exp Clin Psychopharmacol 5:256-262

Mazur JE (2006) Mathematical models and the experimental analysis of behavior. J Exp Anal Behav 85:275-291

McNamara R, Dalley JW, Robbins TW, Everitt BJ, Belin D (2010) Trait-like impulsivity does not predict escalation of heroin selfadministration in the rat. Psychopharmacology (Berl) 212:453464

Mitchell SH (1999) Measures of impulsivity in cigarette smokers and non-smokers. Psychopharmacology (Berl) 146:455-464
Nader K, van der Kooy D (1997) Deprivation state switches the neurobiological substrates mediating opiate reward in the ventral tegmental area. J Neurosci 17:383-390

Odum AL, Madden GJ, Badger GJ, Bickel WK (2000) Needle sharing in opioid-dependent outpatients: psychological processes underlying risk. Drug Alcohol Depend 60:259-266

Pattij T, Vanderschuren LJMJ (2008) The neuropharmacology of impulsive behaviour. Trends Pharmacol Sci 29:192-199

Pattij T, Schetters D, Janssen MC, Wiskerke J, Schoffelmeer AN (2009) Acute effects of morphine on distinct forms of impulsive behavior in rats. Psychopharmacology (Berl) 205:489-502

Perry JL, Carroll ME (2008) The role of impulsive behavior in drug abuse. Psychopharmacology (Berl) 200:1-26

Perry JL, Nelson SE, Carroll ME (2008) Impulsive choice as a predictor of acquisition of IV cocaine self-administration and reinstatement of cocaine-seeking behavior in male and female rats. Exp Clin Psychopharmacol 16:165-177

Pettit HO, Ettenberg A, Bloom FE, Koob GF (1984) Destruction of dopamine in the nucleus accumbens selectively attenuates cocaine but not heroin self-administration in rats. Psychopharmacology (Berl) 84:167-173

Pierce RC, Kumaresan V (2006) The mesolimbic dopamine system: the final common pathway for the reinforcing effect of drugs of abuse? Neurosci Biobehav Rev 30:215-238

Roberts DCS, Bennett SAL (1993) Heroin self-administration in rats under a progressive ratio schedule of reinforcement. Psychopharmacology (Berl) 111:215-218

Van den Oever MC, Goriounova NA, Li KW, Van der Schors RC, Binnekade R, Schoffelmeer AN, Mansvelder HD, Smit AB, Spijker S, De Vries TJ (2008) Prefrontal cortex AMPA receptor plasticity is crucial for cue-induced relapse to heroin-seeking. Nat Neurosci 11:1053-1058

Van den Oever MC, Lubbers BR, Goriounova NA, Li KW, Van der Schors RC, Loos M, Riga D, Wiskerke J, Binnekade R, Stegeman M, Schoffelmeer ANM, Mansvelder HD, Smit AB, De Vries TJ, Spijker S (2010) Extracellular matrix plasticity and GABAergic inhibition of prefrontal cortex pyramidal cells facilitates relapse to heroin seeking. Neuropsychopharmacology 35:2120-2133

van Gaalen MM, van Koten R, Schoffelmeer ANM, Vanderschuren LJMJ (2006) Critical involvement of dopaminergic neurotransmission in impulsive decision making. Biol Psychiatry 60:66-73

Verdejo-García AJ, Lawrence AJ, Clark L (2008) Impulsivity as a vulnerability marker for substance-use disorders: review of findings from high-risk research, problem gamblers and genetic association studies. Neurosci Biobehav Rev 32:777-810

Willuhn I, Wanat MJ, Clark JJ, Phillips PEM (2010) Dopamine signaling in the nucleus accumbens of animals self-administering drugs of abuse. In: Self DW, Staley Gottschalk JK (eds) Behavioral neuroscience of drug addiction. Springer, Berlin, pp 29-71

Wilson JJ (2007) ADHD and substance use disorders: developmental aspects and the impact of stimulant treatment. Am J Addict 16:5-13

Winstanley CA, Eagle DM, Robbins TW (2006) Behavioral models of impulsivity in relation to ADHD: translation between clinical and preclinical studies. Clin Psychol Rev 26:379-395

Winstanley CA, Olausson P, Taylor JR, Jentsch JD (2010) Insight into the relationship between impulsivity and substance abuse from studies using animal models. Alcohol Clin Exp Res 34:1306-1318

Zacny JP, de Wit H (2009) The prescription opioid, oxycodone, does not alter behavioral measures of impulsivity in healthy volunteers. Pharmacol Biochem Behav 94:108-113 